\title{
Influence of Ion Implantation on Magnetic, Structural and Optical Properties of (Ga,Mn)As Epitaxial Films
}

\author{
O. Yastrubchak ${ }^{a, *}$, J.Z. Domagala ${ }^{b}$, J. Sadowski ${ }^{b, c}$, \\ M. $\operatorname{KuLIK}^{a}$, J. ŻUK ${ }^{a}$, R. $\operatorname{SzYMCZAK}^{b}$, A.L. Tóth ${ }^{d}$ \\ AND T. WOSIŃSKI ${ }^{b}$ \\ ${ }^{a}$ Institute of Physics, UMCS \\ pl. Marii Curie-Skłodowskiej 5, 20-031 Lublin, Poland \\ ${ }^{b}$ Institute of Physics, Polish Academy of Sciences, 02-668 Warszawa, Poland \\ ${ }^{c}$ MAX-Lab, Lund University, 22100 Lund, Sweden \\ ${ }^{d}$ Research Institute for Technical Physics and Materials Science \\ Hungarian Academy of Sciences, 1525 Budapest, Hungary
}

We performed implantation experiments, applying both the chemically active oxygen ions and inactive ions of neon noble gas, to thin epitaxial films of $(\mathrm{Ga}, \mathrm{Mn})$ As ferromagnetic semiconductor. Inspection of their magnetic properties by means of a superconducting quantum interference device magnetometer revealed that the implantation with a low dose of either $\mathrm{O}$ or $\mathrm{Ne}$ ions completely suppressed ferromagnetism in the films. Both the high resolution X-ray diffraction technique and the Raman spectroscopy showed significant changes in the structural and optical properties of the films caused by oxygen and neon implantation.

PACS numbers: 75.50.Pp, 61.72.uj, 61.72.Dd, 78.30.Fs

\section{Introduction}

A considerable increase in the research activity developing the basic materials for spin electronics has been observed in recent years. Ferromagnetic semiconductors, such as the thoroughly investigated (Ga,Mn)As, are especially promising as the materials for spintronics since they interrelate both semiconducting and magnetic properties. Extensive research activity aimed at clarifying structural features of thin (Ga,Mn)As films grown on GaAs substrates caused considerable progress in both understanding the physical phenomena leading to ferromagnetism in III-V

*corresponding author; e-mail: yastrub@hektor.umcs.lublin.pl 
ferromagnetic semiconductors and improving their properties [1]. Recent efforts are directed to developing effective methods of modification of magnetic, electrical, optical and structural properties of $(\mathrm{Ga}, \mathrm{Mn})$ As epitaxial films for nanoelectronic applications $[2,3]$.

It has already been shown that the low-energy low-dose oxygen ion implantation destroys both the conductivity and ferromagnetism in (Ga,Mn)As films and was successfully applied for nanostructure tailoring [4]. However, a mechanism responsible for such modification of the film properties has not been resolved so far. In the present work, in order to elucidate this mechanism, we have performed implantation experiments, applying both the chemically active oxygen ions and inactive ions of neon noble gas, to thin $(\mathrm{Ga}, \mathrm{Mn})$ As films.

\section{Experimental}

Ferromagnetic (Ga,Mn)As films with a low Mn content of $1 \%$ have been grown by the low-temperature molecular-beam epitaxy (LT-MBE) method on semi-insulating (001)-oriented GaAs substrates. The films were $50 \mathrm{~nm}$ thick and were covered with a $10 \mathrm{~nm}$ thick GaAs cap layers. The ion implantation experiments have been performed at room temperature using an ion beam tilted by an angle of $8^{\circ}$ in order to avoid the channeling effect. Ion accelerating energies of $25 \mathrm{keV}$ and $35 \mathrm{keV}$ in the case of $\mathrm{O}^{+}$and $\mathrm{Ne}^{+}$ions, respectively, were chosen to assure the implantation range corresponding to the bottom of the (Ga,Mn)As film. Low ion dose of $5 \times 10^{13}$ ions $/ \mathrm{cm}^{2}$ has been applied in both cases, which corresponded roughly to the number of implanted ions of one order on magnitude smaller than the number of $\mathrm{Mn}$ ions in the $\mathrm{Ga}_{0.99} \mathrm{Mn}_{0.01} \mathrm{As}$ film.

A number of complementary characterization techniques have been used with the aim to study an effect of ion implantation on the film properties. Both the spectroscopic ellipsometry (SE), performed in the spectral range of 2.6-4.0 eV, and the Raman spectroscopy, performed under an excitation of the $514 \mathrm{~nm}$ line of an argon laser, were employed to analyze implantation induced changes in optical properties of the $(\mathrm{Ga}, \mathrm{Mn})$ As films. Structural modifications of the films caused by ion implantation were investigated using a high resolution X-ray diffraction (XRD) technique. Magnetic properties of the films were inspected by means of a superconducting quantum interference device (SQUID) magnetometer.

\section{Results and discussion}

SQUID magnetometry applied to the as-grown $\mathrm{Ga}_{0.99} \mathrm{Mn}_{0.01} \mathrm{As}$ film has shown that it exhibits an in-plane easy axis of magnetization and the Curie temperature of $50 \mathrm{~K}$. Figure 1 presents the magnetization hysteresis curve measured at $5 \mathrm{~K}$ under a magnetic field applied parallel to the film plane. A relatively high Curie temperature and a large value of the low-temperature saturation magnetization [5] point to a high quality of the $\mathrm{Ga}_{0.99} \mathrm{Mn}_{0.01}$ As film. SQUID magnetometry examination of the samples subjected to implantation, with both the $\mathrm{O}^{+}$and $\mathrm{Ne}^{+}$ 
ions, revealed only diamagnetic contribution of the GaAs substrate, as shown in Fig. 1 for the $\mathrm{O}^{+}$implanted sample. This is the evidence that the implantation with a low dose of either $\mathrm{O}$ or Ne ions completely suppressed ferromagnetism in the films.

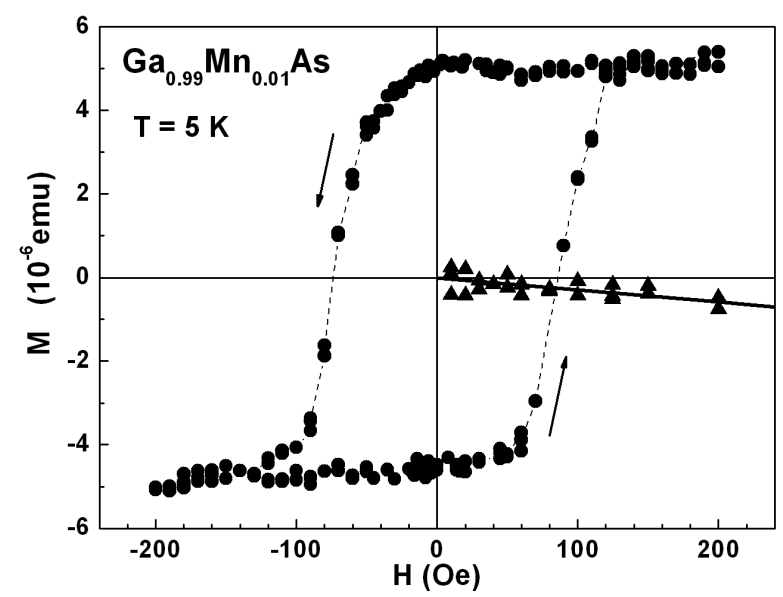

Fig. 1. Magnetization vs. magnetic field dependences measured with a SQUID magnetometer at $5 \mathrm{~K}$ for the as-grown $\mathrm{Ga}_{0.99} \mathrm{Mn}_{0.01} \mathrm{As}$ film, after subtraction of diamagnetic contribution from the GaAs substrate (full circles) and for the sample subjected to oxygen ion implantation (full triangles). The solid line represents the calculated diamagnetic contribution of the GaAs substrate.

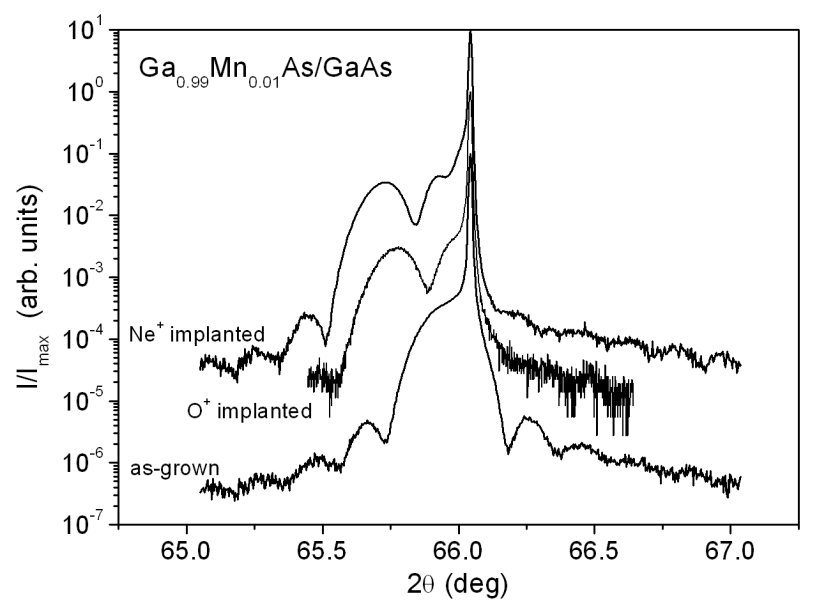

Fig. 2. 004 diffraction curves $(2 \theta / \omega$ scans $)$ for the as-grown and $\mathrm{O}^{+}$and $\mathrm{Ne}^{+}$implanted $\mathrm{Ga}_{0.99} \mathrm{Mn}_{0.01} \mathrm{As} / \mathrm{GaAs}$ heterostructures. The curves have been vertically offset for clarity. The narrow line corresponds to reflection from the GaAs substrate and the broader peaks at lower angles are reflections from the (Ga,Mn)As films. 
The structural quality of the (Ga,Mn)As films was estimated from the XRD results presented in Fig. 2. The clear X-ray interference fringes prove a high-structural perfection of the as-grown $\mathrm{Ga}_{0.99} \mathrm{Mn}_{0.01} \mathrm{As}$ film, which is fully strained to the GaAs substrate. The film thickness calculated from the angular spacing of the fringes corresponds very well to its thickness determined from the growth parameters. Angular positions of the peaks corresponding to the as-grown and implanted films were used to calculate the perpendicular lattice parameters and hence, the vertical lattice mismatch, which was $0.18 \%$ for the as-grown film and, respectively, $0.37 \%$ and $0.43 \%$ for the $\mathrm{O}^{+}$and $\mathrm{Ne}^{+}$implanted films. The larger lattice mismatch revealed in the $\mathrm{Ne}^{+}$implanted film with respect to that in the $\mathrm{O}^{+}$implanted one results likely from the larger ionic radius of neon than that of oxygen.

On the other hand, the results of both the SE (not presented here) and the Raman spectroscopy investigations evidence that $\mathrm{O}^{+}$implantation influences the optical properties of the film significantly stronger than $\mathrm{Ne}^{+}$implantation.

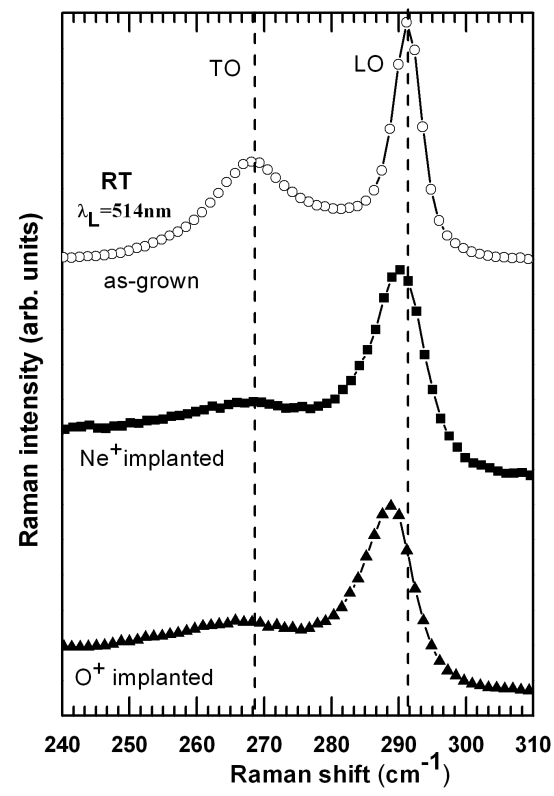

Fig. 3. Micro-Raman spectra recorded at room temperature in backscattering configuration from the (001) surfaces of the as-grown and $\mathrm{Ne}^{+}$and $\mathrm{O}^{+}$implanted $\mathrm{Ga}_{0.99} \mathrm{Mn}_{0.01} \mathrm{As} / \mathrm{GaAs}$ heterostructures. The dashed lines indicate the positions of the Raman LO- and TO-phonon lines for the as-grown heterostructure.

The micro-Raman spectra of the as-grown and implanted samples recorded at room temperature with the light spot diameter of $2 \mu \mathrm{m}$ are presented in Fig. 3 . The spectra were recorded from the (001) surface in the backscattering configuration, where the Raman scattering by longitudinal optical (LO) phonons via 
the deformation potential and the Fröhlich electron-phonon interaction is allowed only. The signal of the symmetry-forbidden transverse optical (TO) phonon originates mainly from disorder scattering and is caused by a slight deviation from (001) surface orientation due to a residual strain in the epitaxial film [6]. The spectra presented in Fig. 3 show that, as a result of the oxygen ion implantation, both the LO-phonon and TO-phonon lines are shifted towards smaller wave number by 3 and $4 \mathrm{~cm}^{-1}$, respectively. Moreover, the amplitudes of TO-phonon lines are distinctly reduced in the implanted samples. This might be caused by crystal structure deterioration owing to a partial strain relaxation in the the implanted films; cf. [7]. This would be in agreement with the XRD results, presented in Fig. 2, showing much weaker X-ray interference fringes for the implanted films.

\section{Conclusions}

SQUID magnetometry investigations proved that the implantation of thin (Ga,Mn)As films with a low dose of either O or Ne ions completely suppressed ferromagnetism in the films. Such an implantation could be applied as a method for tailoring of nanostructures in the films without any post-implantation annealing, which might result in a blurring of the implanted patterns owing to lateral diffusion. The $\mathrm{O}$ and Ne implantation resulted in significant changes of both the structural and optical properties of the films, while the Ne implanted films preserved their higher structural and optical quality. Further studies are needed to clarify the mechanism responsible for suppression of ferromagnetism in (Ga,Mn) As films by ion implantation.

\section{Acknowledgments}

O.Y. acknowledges financial support by the Polish Ministry of Science and Higher Education (MSHE) under grant POL-POSTDOC III, N PBZ/MNiSW/07/2006/33. Ion implantation was performed in the Department of Ion Beam Physics and Implantation, UMCS, Lublin. The work was partly supported by the MSHE project No. N507 461833.

\section{References}

[1] T. Dietl, H. Ohno, F. Matsukura, Phys. Rev. B 63, 195205 (2001).

[2] L. Thevenard, A. Miard, L. Vila, G. Faini, A. Lemaitre, N. Vernier, J. Ferré, S. Fusil, Appl. Phys. Lett. 91, 142511 (2007).

[3] R. Farshchi, R.V. Chopdekar, Y. Suzuki, P.D. Ashby, I.D. Sharp, J.W. Beeman, E.E. Haller, O.D. Dubon, Phys. Status Solidi C 4, 1755 (2007).

[4] T. Figielski, T. Wosiński, A. Morawski, O. Pelya, J. Sadowski, A.L. Tóth, J. Jagielski, Phys. Status Solidi A 195, 228 (2003).

[5] T. Wosinski, T. Figielski, O. Pelya, A. Makosa, A. Morawski, J. Sadowski, W. Dobrowolski, R. Szymczak, J. Wrobel, Phys. Status Solidi A 204, 472 (2007). 
[6] V. Bellani, C. Bocchi, T. Ciabattoni, S. Franchi, P. Frigeri, P. Galinetto, M. Geddo, F. Germini, G. Guizzetti, L. Nasi, M. Patrini, L. Seravalli, G. Trevisi, Eur. Phys. J. B 56, 217 (2007).

[7] O. Brafman, D. Fekete, F.L. Sarfaty, Appl. Phys. Lett. 58, 400 (1991). 